\title{
Sínqapore Journal of Cardíoloxy
}

\section{Original Article}

\section{Burden of Cardiovascular Diseases in the GCC Countries:-Finding from the Global Burden Diseases Study 1990-2017}

\author{
Dr. Sakina Bouzekouk* \\ ${ }^{* 1}$ Department of Internal medicine, Al-Emadi Hospital, North gate, Doha, Qatar \\ *Cardio-mani@hotmail.com
}

Received on: 29-08-2020; Revised and Accepted on: 09-09-2020

\begin{abstract}
Background: The Cardiovascular Diseases (CVD) burden keeps increasing worldwide. GCC countries, especially with the socio-economic development they knew, are not spared from this trend. Nevertheless, an analysis of its time trend profile and its distribution across the entire region GCC is not available in the literature. In this research paper, relying on the reported data from the Global Burden Diseases Study findings between 1990-2017, a detailed analysis of the cardiovascular diseases patterns and their time changing across the GCC region between 1990 and 2017 is presented.

Methods: Based on the available data from the Global Burden of Diseases (GBD) Study 2017, prevalence, mortality and disability adjusted Life-years (DALYS) caused by cardiovascular diseases (CVD) were analyzed. The major causes of deaths due to CVD in the GCC region were analyzed and given a special attention. Discrepancies in CVD disease burden among the different GCC countries were analyzed.

Results: The overall contribution of CVD in GCC countries was estimated to be around 48016.64 cases (95\% UI: 42385.31-54590.44) of the total deaths in the year 2017 equivalent to $31.92 \%$ of the total deaths, while the total DALYs was found to be around 1,457,747.55 (95\% UI: 1,259,653.45-1,687,706.93) in the same year. The change in death percentage and DALY between 1990 and 2017 the percentage were $106 \%$ and $138 \%$, respectively.

The death number caused by ischemic heart disease in the GCC showed an increase from 13443.86 (95\% UI: 11483.96 -15949.11) in 1990 to 31410.25 (95\% UI: 27555.21 - 35928.94) in 2017 corresponding to $57.65 \%$ and $66.78 \%$ of the total CVD deaths. Meanwhile, the number deaths by stroke increased from 7,413.61 (95\% UI: 8,584.72 to 6,363.59) to 11,280.02 (95\% UI: 12,974.16 - 9,830.88) within the same period making a 1.52 times increase approximately.

Out of a total of 48016.64 CVD deaths (95\% UI: 42385.31-54590.44) registered GCC countries in 2017, 31568.6 (95\% UI: 28436.735356.77) deaths were registered in Saudi Arabia making it the highest number and the lowest number of deaths was registered in Qatar 917.57 (95\% UI: 768.17-1081.73). However, the highest percentage was registered in Saudi Arabia with 91.65 per 100,000 capita followed by Oman (91.01 per 100,000 capita) and the lowest registered percentage was in Qatar with 33.4 per 100,000 capita.

Conclusion: The major causes of deaths for CVD diseases in the GCC region were found related to Ischemic heart diseases followed by Stroke which represent together more than 78\% of the total CVD deaths for the year 2017. Compared to the global average, the age standardized DALYs rates in the GCC countries were higher. Therefore, a better comprehensive approach is required to prevent and alleviate the burden of CVD in the region.
\end{abstract}

Keywords: Cardiovascular disease; burden; GCC region; prevalence, death; age standardized

\section{INTRODUCTION:}

Cardiovascular diseases (CVDs) are a major concern for health professionals worldwide as they become the main cause of mortality globally and a major contributor to reduced quality of life. The alarming increase of Non-Communicable Diseases (NCDs) including CVDs, represent a concern for global health and major threats to Sustainable Development Goals in the 21st century as declared by the United Nations (UN) in 2011 [1].

\section{*Corresponding Author:}

Dr. Sakina Bouzekouk

Department of Internal medicine, Al-Emadi Hospital, North gate, Doha, Qatar

Email: Cardio-mani@hotmail.com

DOI: doi.org/10.46978/sjc.20.1.2.15 
Subsequently in 2013, the World Health Organization (WHO) developed clearly defined plans for the prevention and control of NCDs and has set goals to decrease mortality from NCD. Among them, the third sustainable development goal which recognized the importance of CVD by targeting a one-third reduction in premature mortality due to non-communicable diseases [2].

Understanding global trends of the population the health status and the variations with time in the main causes of deaths and disease burden is essential to tracking progress towards the Sustainable Development Goals to ensure healthier societies and improve quality of life for all population at all ages [3]. Assessment of these trends requires well defined objectives and standard measures of population health to help health professionals and policy makers identify priorities and set plans to address rising challenges and achieving the goals. The Global Burden of Diseases, Injuries, and Risk Factors Study (GBD) 2017, uses a combination of up-to-date epidemiological data and improved standardized methods to provide a comparative assessment of health situations for 195 countries and territories. The Global Burden of Diseases, Injuries, and Risk Factors Study (GBD) 2017results provide a completely new mortality time-series of CVD deaths and regional patterns of total CVD mortality estimated from 1990 forward and updated through 2017. GBD 2017 provides a complete reanalysis of all available data by country from 1990 to 2017, and thus supersedes all previously published GBD estimations of HALE and DALYs. According to the Global Burden of Disease (GBD) study published in the year 2017, CVD have been the leading cause of global mortality since 1980 (Institute for Health Metrics and Evaluation; Mortality and Causes of Death 2017). With an estimated 17.8 million deaths, nearly one-third of all deaths worldwide in 2017 were caused by CVD diseases $[4,5]$, representing $31 \%$ of all global deaths. Cardiovascular diseases (CVDs) are a group of disorders of the heart and blood vessels including ischemic heart disease, stroke, heart failure, peripheral arterial disease, and a number of other cardiac and vascular conditions $[4,5]$. Ischemic heart disease and stroke accounted for $85.1 \%$ of all deaths in the CVD category in 2017 [6].

The Gulf Cooperation Council (GCC) is a regional organization comprising Saudi Arabia, Bahrain, Oman, Qatar, the United Arab Emirates, and Kuwait that was created in 1981 to encourage investment and to adopt free trade between member states. With globalization and rapid urbanization of most GCC societies, driven by large exports of oil and natural gas, deep changes in populations lifestyle and habits has occurred, such as large consumption of poor quality foods and the adoption of a sedentary lifestyle [7] that resulted in increased rates of CVD and associated risk factors which, exceed that of developed countries [7]. These facts, let to increased number of deaths resulting from ischemic heart disease and hypertensive heart problems as well as the number of disability-adjusted life years (DALYs) resulting from ischemic and hypertensive heart disease in the region [8]. Although several publications on the prevalence of CVD and/or CVD risk factors in the Middle East are available in the literature [9-11], no recent publications were observed specifically for the GCC countries. The aim of this paper is to analyze the CVD burden in GCC countries, among the adult population in the GCC Based on the findings on CVD between 1990 and 2017, from the GBD, Injuries and Risk Factors Study (GBD 2017) for the six GCC countries.

\section{METHODS}

\subsection{GBD Estimation Framework}

The CVD burden data employed in this work were extracted from the GBD study for the year 2017. The GBD study comprehensively and systematically quantifies the comparative magnitude of health loss over time due to 315 diseases and injuries 2619 unique sequelae, and 84 risk factors by age, sex, and location for 195 countries, 21 regions, and seven super-regions from 1990 to 2017 Detailed methodology approach of GBD 2017 and specific methodology used for CVD are available in (GBD 2017 Disability-adjusted Life Years (DALYs) and Collaborators 2017; GBD 2017 Disease and Injury Prevalence Collaborators 2017; GBD 2017 Mortality and Causes of Death Collaborators 2017).

\subsection{Defining Disease Categories}

CVD category includes the 10 most common global causes of CVD-related death. These causes were ischemic heart disease (IHD), ischemic stroke, hemorrhagic and other stroke, atrial fibrillation, peripheral arterial disease (PAD), aortic aneurysm, cardiomyopathy and myocarditis, hypertensive heart disease, endocarditis, rheumatic heart disease (RHD), and a category for other CVD conditions.

\subsection{Data Sources And Analytic Methods}

Data on CVDs burden and risk factors were retrieved from the Global Health Data Exchange of the Institute for Health Metrics and Evaluation (IHME) map of data, available for each country at the University of Washington http://www.healthdata.org/. GBD is a global comparative risk assessment exercise, with the first preliminary results for base year 1990 published in the World Development Report 1993[12]. Detailed descriptions of the IHME's data collection methodology have been published previously $[13,14]$. Although most of the information needed are available from the IHME website, this information needs to be filtered and interpreted in a coherent, consistent way, for its presentation and eventual use and implementation, at its global, regional, national and other granularity levels.

\subsection{Disability-Adjusted Life Years}

Disability adjusted life-years (DALYs) combine information regarding premature death (years of life lost [YLL]) and disability caused by the condition (years lived with disability [YLD]) to provide a summary measure of health lost due to that 
condition. YLL was calculated by multiplying observed deaths for a specific age in the year of interest by the age specific reference life expectancy estimated using life table methods. YLD was calculated by multiplying disease prevalence by a health-state-specific disability weight representing a degree of lost functional capacity. A detailed explanation of the process of disability weight estimation has been reported separately $[15,16]$.

\section{RESULTS}

Results of the GBD 2017 study, including prevalence, prevalence, mortality, and DALYs, for all GCC countries for the years 1990-2017 were obtained from the GBD results using the interactive webpage (22).

\subsection{Prevalence}

It was estimated that $2,473,344.85$ prevalent cases of CVD (95\% UI: 2,337,198.98 - 2,622,431.53) in 2017 were registered in the GCC countries. Out of them 1,579,061.86 (95\% UI: 1,490,977.93-674,220.09) representing $64 \%$ of the total prevalent cases were males while $36 \%$ were made of females.

The age-standardized prevalence of CVD by country varied slightly (Figure 1) with the lowest average age-standardized observed in UAE. However, there was an increase in the agestandardized prevalence of CVD over the years from 1990 to 2017 in all GCC countries. The lowest age-standardized prevalence in 2017 was registered in Qatar while the highest age-standardized prevalence was registered in Saudi Arabia.

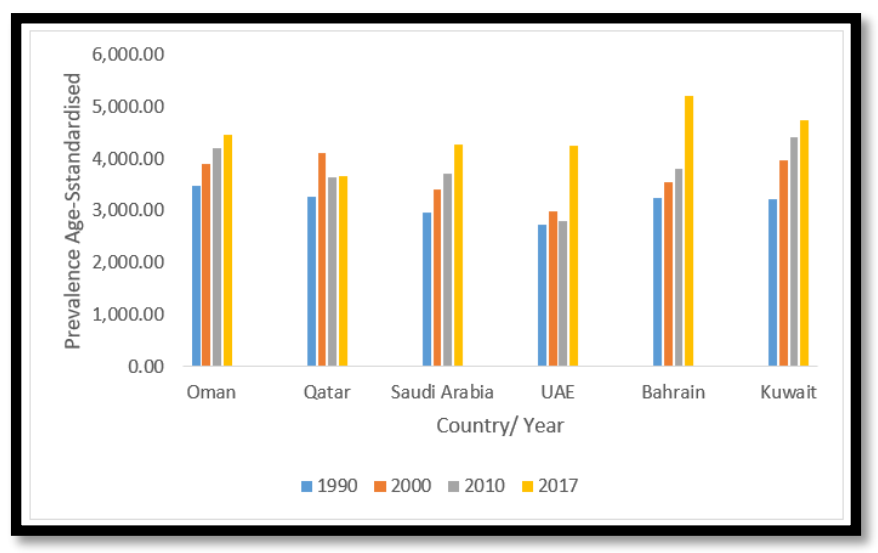

Fig 1: Prevalence Age-Standardized Rates from 1990 to 2017in GCC countries

\subsection{Mortality}

It was estimated that about 48016.64 cases of CVD deaths (95\% UI: 42385.31 - 54590.44 cases) were registered in the GCC countries in 2017. The age-standardized prevalence of CVD varied significantly by country as shown in Figure 1. Qatar has the lowest age-standardized prevalence in 2017 of 33.4, while Saudi Arabia registered the highest age-standardized prevalence in 2017 of 91.65 per 100,000 persons. Qatar also registered the lowest age-standardized prevalence for both male and females with 27.4 and 35.35 per 100,000 persons respectively. The highest age-standardized prevalence for female of 94.4 was registered in Oman against 104.84 for males registered in Saudi Arabia. In general, the age-standardized prevalence for females is lower than that of males except in Oman where the opposite behavior was observed.

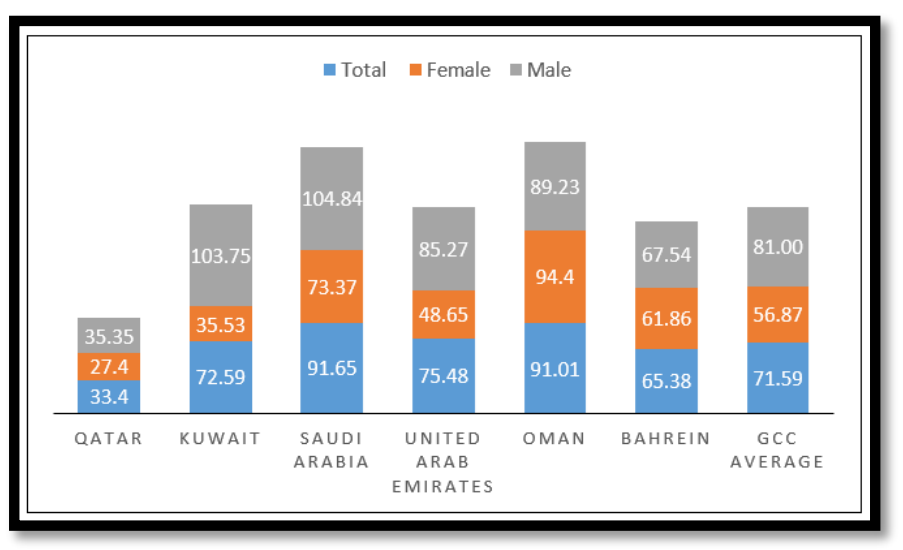

Fig 2: Average Age-Standardized Death Rates in 2017, by Sex of CVD Mortality

There were 23316.14 deaths in GCC due to CVD in 1990, increasing to 48016.64 deaths in 2017 as shown in with an average change percentage of $106 \%$ for all GCC countries. However, the highest percentage of change (507\%) was observed in UAE while the lowest change percentage of $30 \%$ was registered in Bahrein. These deaths accounted for about $32 \%$ of all deaths in the region in 2017 , compared to $29 \%$ of all deaths in 1990.

There was broad variation in the age-standardized CVD mortality rate among GCC countries (Figure 2) .Significant decline in the age-standardized death rate due to CVD occurred between 1990 and 2017 in Qatar, Oman and Bahrein. However, no significant changes were detected over this time period for Kuwait and Saudi Arabia. UAE had a small increase in the agestandardized death rate due to CVD.

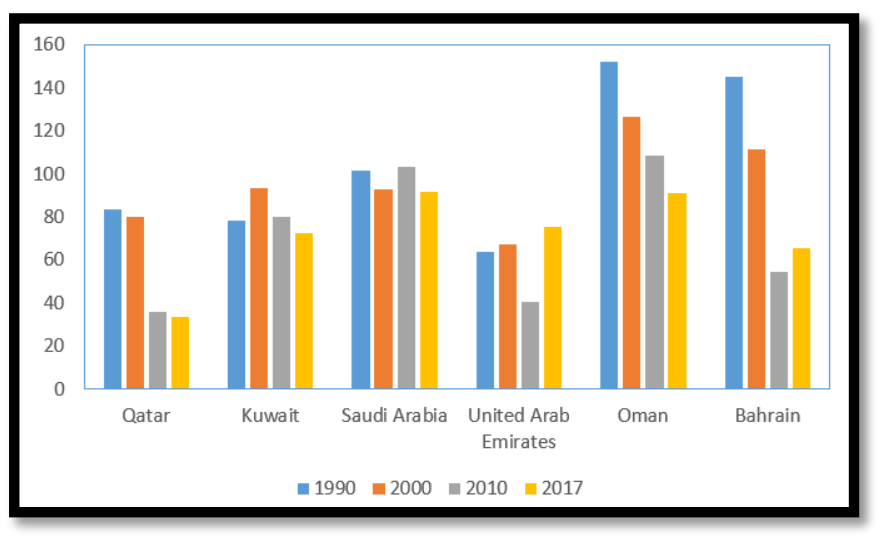

Fig 2: Variation of Age standardized deaths (per100, 000) through 1990-2017 in GCC countries 


\subsection{Major CVD deaths categories}

Figure 3 shows the total number of deaths from all CVD categories mounting to a total of 41828.02 deaths in all GCC countries in 2017. Ischemic Heart Disease (IHD) deaths were 31410.25 which accounted for $66.78 \%$ of the total number of deaths due to CVD in the GCC region, followed by Ischemic Stroke (11.43\%), Hemorrhagic stroke 11.07\%, Hypertensive heart diseases (2.95\%) and Myocarditis (2.75\%) . other uncategorized diseases mounted for $2.98 \%$ of the total CVD deaths. A graphical representation of all CVD disease distribution is shown in Figure 3. IHD and stroke mortality rates is the main cause of CVD deaths in all GCC countries, supporting the theory of an epidemiological transition for CVD $[17,18]$.

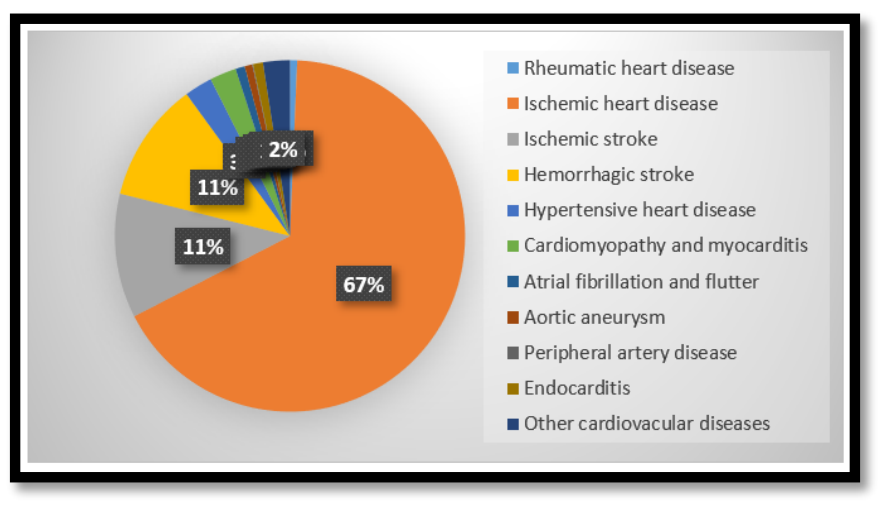

Fig 3: Distribution of Major CVD disease categories in GCC countries in 2017.

\subsection{DALYs}

The number of disability-adjusted life years (DALYs) of CVD for different GGC countries from 1990 to 2017 is presented in Figure 4. The number of DALYs from CVD increased from $613,671.86$ in 1990 to $1,457,747.55$ in 2017, making an increase of $138 \%$. As shown in Figure 4, DALY numbers increased in all GCC countries from 1990 to 2017 but with different rates; the highest increase rates were observed in UAE followed by Qatar. The lowest rates changes were observed in Bahrein and Oman.

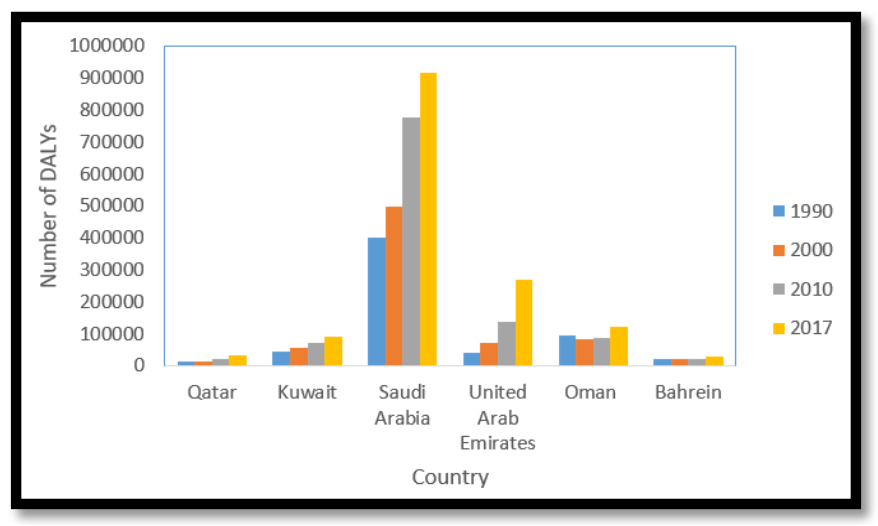

Fig 4: Number of DALY for CVD causes of death from 1990 to 2017 in GCC countries

\section{DISCUSSION}

Cardiovascular diseases are the leading cause of disease burden in GCC countries. According to the GBD 2017, the total number of CVD deaths in the GCC increased with a percentage change of $106 \%$ from 23,316.14 (95\% UI: 20,414.90$27,004.50$ ) in 1990 to $48,016.64$ cases (95\% UI: $42385.31-$ 54590.44 ) in 2017. These deaths accounted for $29.05 \%$ of all deaths in the region in 1990 compared to $31.92 \%$ of all deaths in 2017. Ischemic Heart Disease (IHD) was 31410.25 (95\% UI: 27555.21 - 35928.94) in 2017, which accounted for $66.78 \%$ of the total number of deaths due to CVD in the GCC region.

The age-standardized prevalence of CVD varies slightly from a country to another. In year 2017, the lowest age-standardized prevalence was registered in Qatar with 3671.62 cases (per 100,000 ) while the highest age-standardized prevalence was observed in Saudi Arabia with 5217.77 (per 100K). However, there was an increase in the age-standardized prevalence of CVD over the years from 1990 to 2017 in all GCC countries.

The number of DALYs from CVD increased from 613,671.86 (95\% UI: 537,833.78 - 702,899.73) in 1990 and 1,457,747.55 (95\% UI: $1,259,653.45-1,687,706.93$ ) in 2017 , a $138 \%$ increase. DALY number increased in all GCC countries from 1990 to 2017; the highest increase in DALY number was seen in UAE (558\%) followed by Qatar (161\%), Saudi Arabia (128\%) and Kuwait (110\%). The lowest DALY numbers were registered in Bahrein (41\%) and Oman (28\%).

Based on the findings of this work, especially on CVD mortality data in 2017, it is expected that the GCC countries will not be able to achieve the target of $25 \%$ reduction of CVD mortality by 2025 unless drastic measures and new stringent policies will be taken. Moreover, it is recommended that some of the GCC countries reemphasize the need for additional investments and improvements in the health system management in general with a particular attention to CVD burden. A comprehensive approach for monitoring preventing and controlling the burden of CVD in GCC countries is required. It is recommended that educational programs to increase awareness among the population on the importance of controlling CVD risk factors should be implemented.

\section{CONCLUSION}

The rapid socioeconomic growth of most GCC societies caused deep changes in the population's lifestyle and habit such as consumption of poor quality foods and the adoption of a sedentary lifestyle resulted in increased number of deaths caused by ischemic heart disease and hypertensive heart problems. Implementing stringent programs to reduce the burden of cardiovascular diseases becomes an urgent matter. This burden will further increase with aging and growth of the population unless effective measures will be taken in the near future. Hence, a comprehensive approach to monitor, prevent 
and control the burden of CVD in the region should be considered.

\section{REFERENCE}

1. Alwan, A., Global status report on noncommunicable diseases 2010. 2011: World Health Organization.

2. Reddy, K.S., Global Burden of Disease Study 2015 provides GPS for global health 2030. The Lancet, 2016. 388(10053): p. 1448-1449.

3. Organization, W.H., SDG3 and beyond: healthier environments for healthier populations in the Sustainable Development Goals. 2019.

4. Kyu, H.H., et al., Global, regional, and national disabilityadjusted life-years (DALYs) for 359 diseases and injuries and healthy life expectancy (HALE) for 195 countries and territories, 1990-2017: a systematic analysis for the Global Burden of Disease Study 2017. 2018. 392(10159): p. 18591922.

5. Collaborators, G., et al., Global, regional, and national agesex-specific mortality for 282 causes of death in 195 countries and territories, 1980-2017: a systematic analysis for the Global Burden of Disease Study 2017. 2018. 392(10159): p. 1736-1788.

6. Roth, G.A., et al., Global, regional, and national age-sexspecific mortality for 282 causes of death in 195 countries and territories, 1980-2017: a systematic analysis for the Global Burden of Disease Study 2017. The Lancet, 2018. 392(10159): p. $1736-1788$.

7. Mabry, R., et al., Evidence of physical activity participation among men and women in the countries of the Gulf Cooperation Council: a review. 2010. 11(6): p. 457-464.

8. Lawes, C.M., S. Vander Hoorn, and A.J.T.L. Rodgers, Global burden of blood-pressure-related disease, 2001. 2008. 371(9623): p. 1513-1518.

9. Motlagh, B., et al., Prevalence of cardiovascular risk factors in the Middle East: a systematic review. 2009. 16(3): p. 268280 .

10. Shara, N.J.N., Metabolism and C. Diseases, Cardiovascular disease in Middle Eastern women. 2010. 20(6): p. 412-418.

11. Aljefree, N. and F. Ahmed, Prevalence of Cardiovascular Disease and Associated Risk Factors among Adult Population in the Gulf Region: A Systematic Review. Advances in Public Health, 2015. 2015: p. 235101.
12. Lea, R.A., World Development Report 1993:'Investing in Health'. 1993.

13. Naghavi, M., et al., Global, regional, and national age-sex specific mortality for 264 causes of death, 1980-2016: a systematic analysis for the Global Burden of Disease Study 2016. 2017. 390(10100): p. 1151-1210.

14. Vos, T., et al., Global, regional, and national incidence, prevalence, and years lived with disability for 328 diseases and injuries for 195 countries, 1990-2016: a systematic analysis for the Global Burden of Disease Study 2016. 2017. 390(10100): p. 1211-1259.

15. Vos, T., et al., Global, regional, and national incidence, prevalence, and years lived with disability for 310 diseases and injuries, 1990-2015: a systematic analysis for the Global Burden of Disease Study 2015. 2016. 388(10053): p. 15451602.

16. Kassebaum, N.J., et al., Global, regional, and national disability-adjusted life-years (DALYs) for 315 diseases and injuries and healthy life expectancy (HALE), 1990-2015: a systematic analysis for the Global Burden of Disease Study 2015. 2016. 388(10053): p. 1603-1658.

17. Abdel, O.J.M.M.F.Q., The epidemiologic transition: a theory of the epidemiology of population change. 1971. 49(4): p. 509538.

18. Olshansky, S.J. and A.B.J.T.M.Q. Ault, The fourth stage of the epidemiologic transition: the age of delayed degenerative diseases. 1986: p. 355-391.

\author{
Article Citation: \\ Authors Name. Sakina Bouzekouk. Burden of Cardiovascular Diseases in the GCC Countries:-Finding from the Global Burden \\ Diseases Study 1990-2017. SJC 2020; 1(3): 90 - 94. \\ DOI: doi.org/10.46978/sjc.20.1.2.15
}

